\title{
Portable four-choice reaction time test with magnetic tape memory
}

\author{
ROBERT T. WILKINSON and DAVID HOUGHTON \\ Medical Research Council, Applied Psychology Unit [Annexe], Cambridge, England
}

\begin{abstract}
A small $(20 \times 11 \times 6 \mathrm{~cm})$, battery-powered, fully portable device is described for administering a four-choice serial reaction time test and recording the results on a standard magnetic tape cassette. The mains-powered decoder through which the tape is subsequently replayed is also described. The main application is the self-administration of the test by the subject in the field, particularly in studies of environmental stress, the data-bearing cassettes being passed to the experimenter for laboratory-based analysis. In preliminary performance trials, the four-choice test appeared to reflect fatigue due to continuous repetitive responding in a way similar to classical, nonportable, multiple-choice serial reaction tests, but over a shorter time scale and with greater internal consistency.
\end{abstract}

Multiple-choice serial reaction time tests, for example Leonard's five-choice test (Leonard, Note 1), have proved sensitive indices of the effects of environmental stress (for review, see Wilkinson, 1969). The tests themselves are usually laboratorybased, because the hardware is relatively bulky, requires mains (line voltage) electricity, and needs a separate store for the data, usually a tape recorder or computer. The object of the present design was to produce a portable version of the choice serial reaction test by greatly reducing the weight and size, by using batteries as a source of power, and by storing a record of the speed and accuracy of responses within the machine for subsequent retrieval using a mains-powered four-choice decoder. The overall aim was that subjects should be able to carry out the test in the field and. if necessary. in the absence of the experimenter.

In order to reduce costs, one of a popular range of battery cassette tape recorders was adapted to perform the triple function of housing the display and response apparatus, generating a program of stimuli, and recording the response data. A laboratory-based decoder was also constructed. When the data are replayed through the decoder, the original sequence of responses is reproduced auditorially in real time. Simultaneously, output pulses are available to indicate the "correct" and "error" responses. These may be used with external equipment for counting or measuring reaction time of responses or as an interface to a general-purpose computer.

\section{GENERAL DESCRIPTION AND MODE OF OPERATION}

\section{Recorder}

The recorder chosen was the Philips EL3302. It is

Requests for reprints should be sent to the senior author at 5 Shattesbury Road. Cambridge, England; technical enquiries about electronic design should be sent to the junior author. shown in its modified form in Figure 1. The display consists of four lights (light-emitting diodes, or LEDs) mounted in the form of a square. Close to these are mounted four push buttons correspondingly arranged to form a square. To administer the test, first switch on the test switch (switch A, Figure 1). Then move forward the transport control (B) in the direction of the single arrow. This will initiate tape transport and recording. One of the lights will now be on, and the test can commence at any time. The subject starts the test by pressing the button corresponding geometrically to the light that is on. The light is extinguished and, after $120 \mathrm{msec}$, either the same light or one of the others comes on, according to a random program of onset. The subject responds appropriately to this and so brings on the next light in the sequence, and so on. He continues to respond in this way as rapidly and accurately as he can for the required period of the test. This will typically vary from 1 to $30 \mathrm{~min}$, as conditions permit. When the subject makes a "correct" response to the light, that is. when the button is pressed which corresponds geometrically to the light that is on, a $2-\mathrm{kHz}$ tone is recorded. A 4-kHz tone records an "error."

Before starting the test, it may be necessary to record details of the test session on the tape, or at least present some indication that it is about to start. For this purpose, the marker button, labeled $C$ in Figure 1 , is pressed. Whenever this is done, a $1-\mathrm{kHz}$ marker tone will be recorded on the tape. The message may vary from a simple pulse to indicate the start and end of the test to messages concerning time, conditions of testing, and so on, relayed in Morse or some simpler code.

\section{Decoder}

To decode a cassette, the experimenter plugs the DIN plug of the decoder into the recorder. The plug enables the recorder to operate from the decoder power supply, thus conserving the batteries. Also, it 


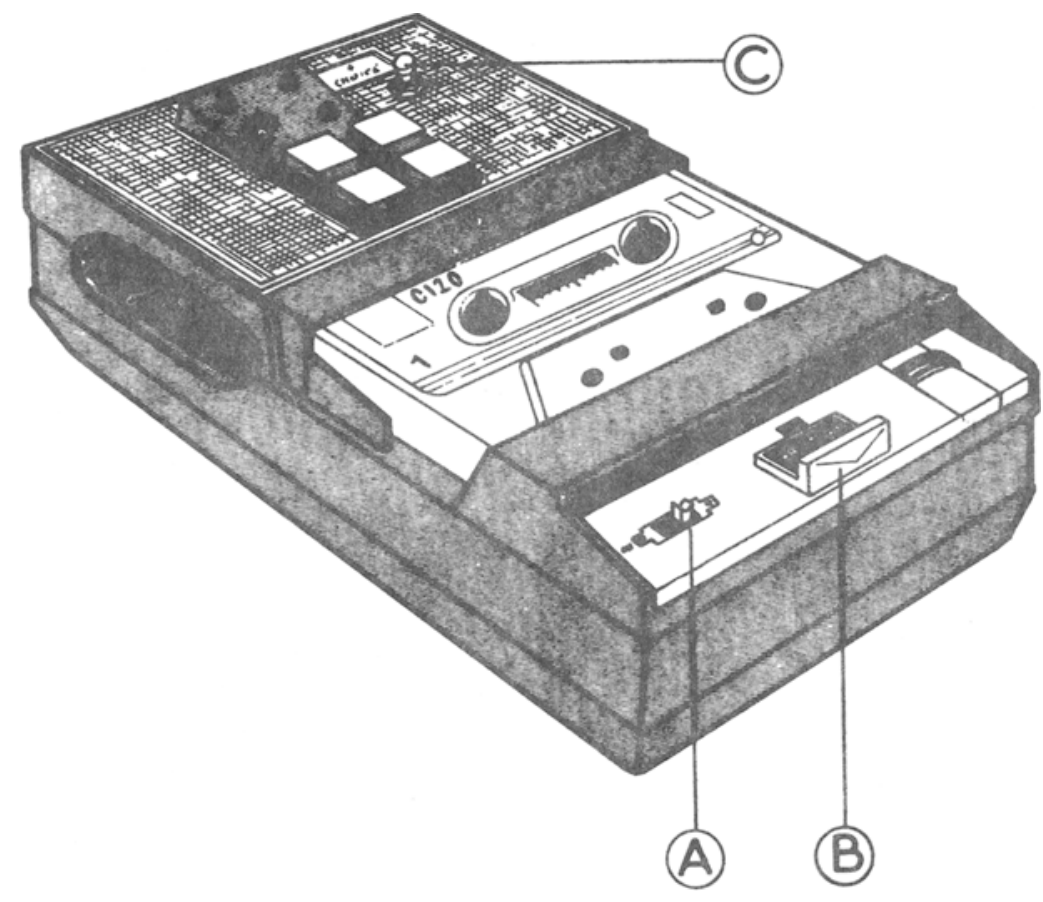

Figure 1. The four-choice reaction time recorder.

takes the signal from the tape for decoding. The tape is rewound and replayed using only the transport control (B, Figure 1). It is emphasized that the tape must not be rewound or replayed with the test switch (A, Figure 1) on as this would mar the recording. The decoder has a volume control which allows the level of the replayed stimuli to be set without affecting the decoding of the tones. A miniature socket gives access to the decoder output. The connections provided are (1) 0 V line, (2) stimulus pulse, (3) "correct" pulse, (4) "error" pulse, (5) "correct" OR "error" pulse, and (6) marker pulse. The active outputs are normally at $0 \mathrm{~V}$ and give a $7 \frac{1}{2}-\mathrm{V}$ positive pulse when the respective signal occurs.

The original tape-recorder printed circuit board and loudspeaker have to be removed to allow space for the modifications. The motor-speed-control 1-in.-sq circuit board, however, is left undisturbed. If it is required to examine the data auditorially in the field, an external replay amplifier and speaker can be connected to the DIN socket of the tape recorder. The tape is then rewound and replayed as described above. Alternatively, a second, conventional, tape recorder may be used.

In order to achieve optimum accuracy of replayed times, the same recorder should be used for replaying the tape as was used for recording. This obviates the effects of slightly different speeds between instruments. Philips' wow and flutter figure (i.e., speed variation) for this instrument is $\leqslant \pm 0.4 \%$.

\section{Erasure}

The original erasing circuit is on the removed circuit board, while the erase head is left in the recorder. Recorded cassettes must therefore be cleared by a bulk eraser if it is intended to use them again. Alternatively, a conventional tape recorder may be used.

\section{RECORDER CIRCUIT DESIGN}

\section{General}

The EL 3302 tape recorder operates on five $11 / 2-\mathrm{V}$ cells, giving a nominal voltage of $71 / 2 \mathrm{~V}$. This supply is also used for the logic circuitry which is powered via the switch $A$ in Figure 1. The condition of the batteries is indicated by the battery level meter, which is reconnected as shown in Figure 2. Details of the cell types and the use of the meter for battery checking are given in the recorder handbook.

\section{Random Light Selector}

An RCA CD4042AE quad D-type latch integrated circuit (IC) is used as a four-stage oscillator. A capacitor-resistor time constant is introduced between each latch, and the clock input is wired so that each latch is active. When power is applied to the circuit, TR2 (Figure 2) instantaneously brings on ( $\bar{Q}$ output at 0 ) one of the four D-latches. It stays on for a period of about $9 \mu \mathrm{sec}$, determined by the resistor and capacitor at its input, $100 \mathrm{k}$ ohms and $100 \mathrm{pF}$, respectively, and the switching level of the logic. On going off, it brings on the next latch for about $9 \mu \mathrm{sec}$, followed by the third, the fourth, and then the first latch again. Thus the $\bar{Q}$ output of only one of the four latches is at logic 0 at any time, and this state is continuously transferred from latch to latch every $9 \mu \sec$ (Figure 3a). 


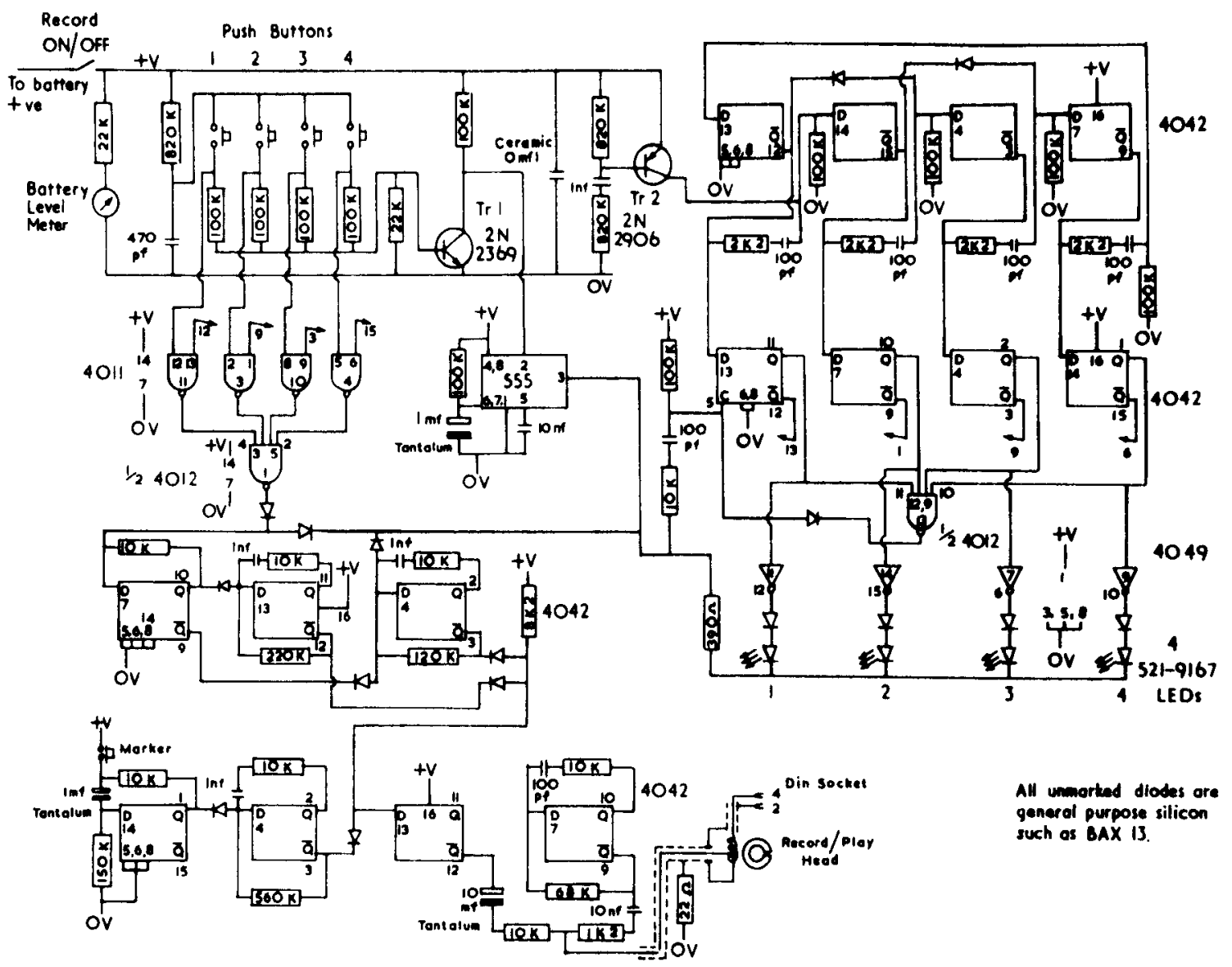

Figure 2. Circuit dlagram of the four-choice test. See Footnote 1 for explanation of expreasions "nf" and 2K2."

All the $Q$ outputs from these latches go to the inputs of a second quad latch shown underneath the first one in Figure 2. The clock input of this IC is connected to be brietly in the clock condition when the NE555V timer pulse ends $120 \mathrm{msec}$ after the subject has pressed a response button (Figure $3 \mathrm{e}$ ). In this manner, the conditions of the $\bar{Q}$ outputs of the lamp-selecting oscillator are sampled by the second set of latches, and the following lamp is selected for illumination. Apart from going to the CD4049AE, which is used to drive the LEDs, the $Q$ outputs of this quad latch go to the four inputs of $1 / 2$ CD4012AE, a four-input NAND gate. The output of this gate will clock the quad latch in the event that it settles in a condition which brings no lamp on. Not until a lamp is selected will the clock pulse be removed.

The four $\bar{Q}$ outputs of the first quad latch or lamp-selecting oscillator are checked with a high-impedance oscilloscope to see that they are all of approximately the same duration (Figure $3 a$ ). In the six prototype units built so far, the output pulses have been practically identical in each individual unit without any adjustment. Where required, the pulse duration from a particular latch can be varied proportionally by changing the value of the $100 \mathrm{kohm}$ resistor at the input of that latch. Thus, this system of lamp selection is designed so that different lamp onset probabilities may be obtained if required.
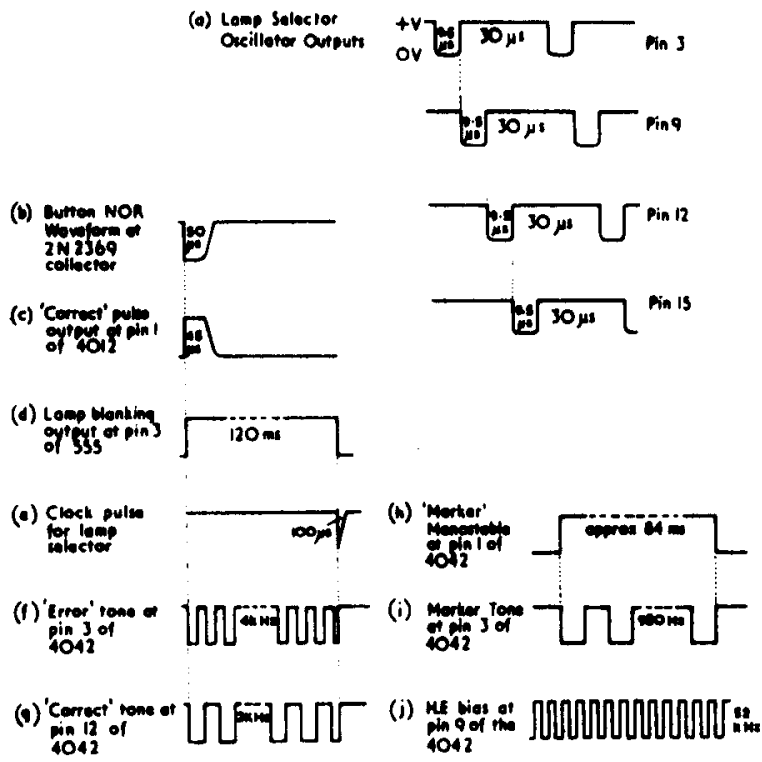

Figure 3. Logic waveforms. 


\section{Lamp Blanking}

Due to the fact that the same lamp may come on several times in succession, a lamp blanking circuit is made to turn the lamps out for a 120 -msec period (Figure 3d), so that each time the subject makes a response he knows he has pressed the button properly. The IC used for blanking the lamps is Signetics NE555V. It is put into its timing mode each time the collector of TR1 goes negative (Figure $3 b$ ) due to a response button's being pressed. The period of this circuit is made as close to $120 \mathrm{msec}$ as possible by selecting a suitable value of resistor. For example, in the units so far constructed, a $12 \mathrm{k} \mathrm{ohm}$ resistor has to be added to the $100 \mathrm{k}$ ohm one shown between Pins 4,8 , and 6,7 .

\section{Scoring and Marker Logic}

When a "correct" button is pressed, both inputs of the corresponding NAND gate of the CD4011AE IC are at logic 1 , and the output is 0 . Thus, one of the inputs of the $1 / 2 \mathrm{CD} 4012 \mathrm{AE}$ four-input gate, which are generally all at 1 , is made 0 and its output changes to 1 for a short duration (Figure 3c). In turn, this triggers the bistable made from one latch in the third CD4042AE. The D input to this latch is Pin 7. Normally, the diode at the $Q$ output of this latch clamps the D input, Pin 13, of a second latch in the same IC at logic 0 . However, when triggered by a "correct" response, the bistable stops clamping until the end of the pulse from the NE555V resets it. In turn, this allows the second latch, which is connected as an astable multivibrator to oscillate at $2 \mathrm{kHz}$ (Figure $3 \mathrm{~g}$ ), for the period of the NE555V pulse. This tone is recorded'on the tape.

The "error" tone generator is made from the third latch of this CD4042AE. Its feedback capacitor is $1 \mathrm{nF}(\mathrm{nF}=1,000 \mathrm{pF})$, and its feedback resistor is $120 \mathrm{k}$ ohms. These values cause it to oscillate at approximately $4 \mathrm{kHz}$. The oscillator is normally clamped at its input, Pin 4 of the IC, by a diode from the NE555V output. However, when any button is pressed, this clamping is removed for $120 \mathrm{msec}$ by the positive pulse from the NE555V output. If the response was a "correct" one, the other diode connected to the above pin, 4 , will take over the clamping requirement. Otherwise, in the event of an "error" response, clamping is completely removed and the oscillator is permitted to record a $4 \cdot \mathrm{kHz}$ tone (Figure $3 \mathrm{f}$ ) on the tape to represent an "error."

The $1-\mathrm{kHz}$ marker tone (Figure $3 \mathrm{i}$ ) is generated in a similar way by two D-latches in a fourth CD4042AE. In this circuit, the duration of the marker tone (Figure 3h) is fixed for the purpose of automatic decoding. However, it could easily be made to have a duration determined by the time for which the marker button is depressed. Then information about the conditions of the test could be put on the tape in a code such as Morse code, in which case decoding would be done by ear.
All the tones go to a diode OR gate and then to a further D-latch used as a buffer stage before being mixed with a high-frequency bias of nominally $56 \mathrm{kHz}$ (Figure $3 \mathrm{j}$ ). This is generated by the fourth D-latch in the fourth CD4042AE IC, whose D input is Pin 7. The mixing of tone and bias is applied to the low-impedance connection of the record/play head. The tones should be adjusted to be within $\pm 10 \%$ of their nominal frequency so that they can readily be decoded. Adjustment is achieved by varying the feedback resistors between $\bar{Q}$ output and $D$ input.

\section{RECORDER CONSTRUCTION}

Hamlin key switches ${ }^{2}$ are mounted on a subchassis consisting of a $45-\mathrm{mm}$ square of $1.6-\mathrm{mm}$ aluminum. The whole assembly is mounted by screws onto the recorder chassis, a hole being made at each corner of the subchassis for the purpose. The LEDs forming the lamp display project slightly from a $35-\mathrm{mm}$ square of 3-mm black Perspex. They are fixed in position by silicone rubber adhesive. The display panel is nıounted alongside the push button subchassis by means of a bracket between the two. The complete subassembly occupies the space vacated by the loudspeaker. Two circuit boards are used and are mounted in sandwich fashion in the position occupied by the original board.

\section{RECORDER COMPONENTS}

The cost of components is about $£ 35$ (about $\$ 80$ ), while the time of construction is $\mathbf{4 0}$ to 80 man-hours. A printed circuit board, as compared with a wired circuit board, would considerably increase the cost but would reduce the construction time. Resistors are $5 \% 1 / 4-W$ and the capacitors mainly $21 / 2 \%$ polystyrene, except for those marked otherwise and the two $10-\mathrm{nF}$ capacitors, which are polyester $2 \%$.

\section{DECODER CIRCUIT DESIGN}

The circuit diagram (Figure 4) shows that the amplifier and loudspeaker extracted from the tape recorder are used for listening to the tones when replaying a cassette. The signal for the decoding logic is taken from the collector of the transistor prior to the volume control on the ex-tape-recorder circuit board. It is amplified further by a single transistor stage, and then transformed by the 741 operational amplifier Schmitt trigger into a square wave. This is passed to the three CD4046AE ICs. Each of these ICs, together with two gates of a CD4001AE, forms a frequency-sensitive switch. Due to the tolerance of the CD4046AE ICs, the resistors connected to Pins 11 and 12 have to be selected to achieve the required frequency capture range. A range of $f \pm 15 \%$ is 


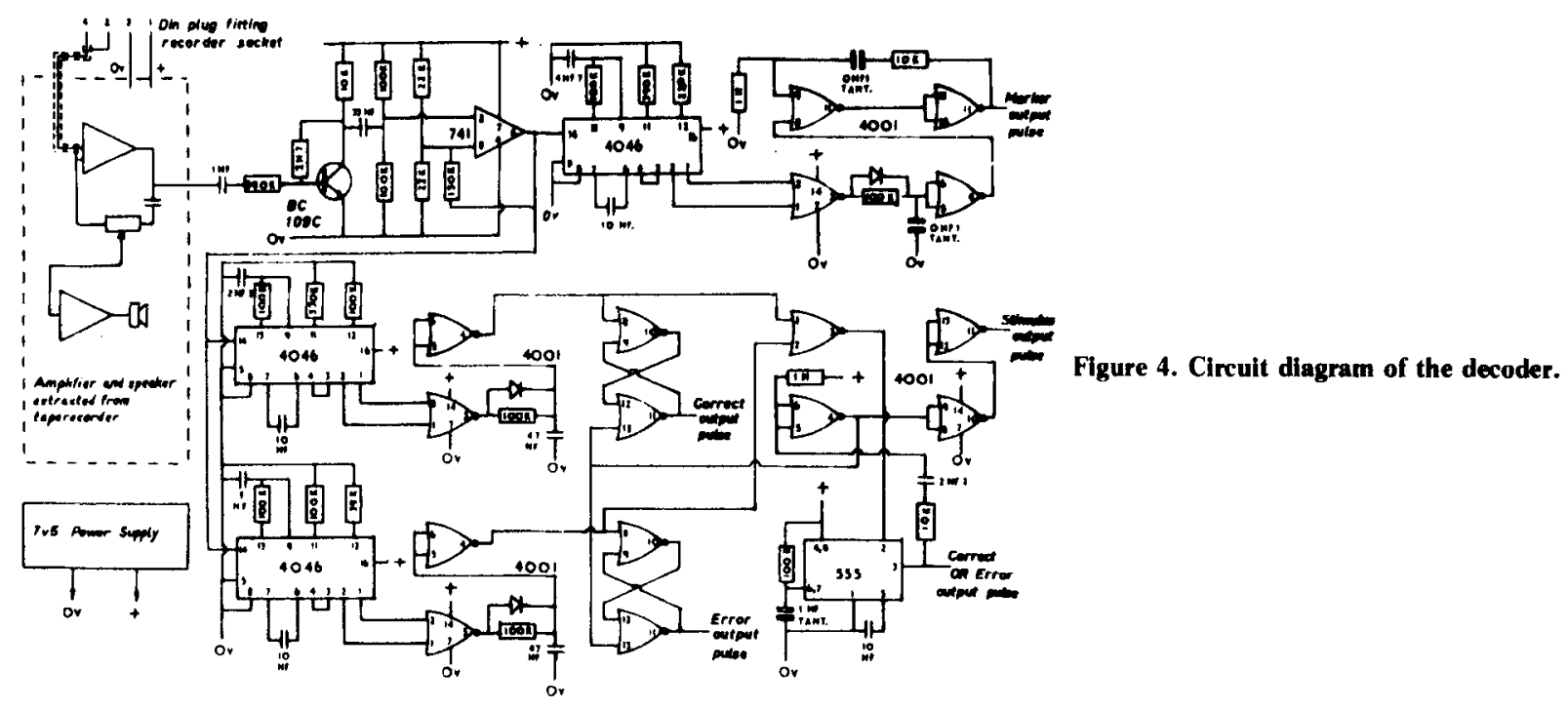

allowed for each of the three tones. The resistor connected to Pin 12 sets the minimum frequency, while that connected to $P$ in 11 sets the capture range. As an example, assume the marker-tone frequencysensitive switch is to be set. The 33-nF capacitor at the input of the 741 Schmitt is connected to a signal generator giving a 4-V peak-to-peak sine-wave output, and an oscilloscope is connected to the output of the second NOR gate. Pin 4. As the generator frequency is raised slowly through $1 \mathrm{kHz}$, the frequency when the NOR gate output goes from 0 to 1 is noted. If this frequency is above $850 \mathrm{~Hz}$, the resistor at Pin 12 needs to be increased, and vice versa. The frequency is then increased until the NOR gate output goes from 1 to 0 . If the frequency is above $1.15 \mathrm{kHz}$, the resister at Pin 11 must be increased. A similar procedure is adopted for the other two frequencysensitive switches. Once these adjustments have been carried out, the power supply voltage must not be changed as the frequencies are sensitive to supply voltage variation.

The output of the marker frequency-sensitive switch operates a monostable multivibrator made from the remaining two NOR gates of a CD4001AE. The monostable obviates the chance of multiple output pulses occurring if a tone is interrupted due to tape dropout. To avoid drop-out effects in the case of the "correct" and "error" tones, the frequencysensitive switches set bistable multivibrators to give a 1 at the output when the appropriate tone occurs. The bistables are made from the remaining gates of the CD4001AEs used with the frequency-sensitive switches. The outputs of the two frequency-sensitive switches go also to a NOR gate, the output of which triggers an NE555V IC timer to give a $120-\mathrm{msec}$ output pulse. The timer is accurately set in the same manner as the one in the recorder. The output from this passes via a time constant to a NOR gate used as an inverter in order to produce a short pulse at the end of the 120 -msec period. This resets the "correct" or "error" bistable ready for the next tone as well as passing via two further gates, used as buffer amplifiers, to give a pulse which indicates onset of stimulus.

\section{DECODER CONSTRUCTION}

The constructional considerations are different from those for the recorder because space and power consumption are not restricted to the same low degree. The critical part is that of mains wiring, which should be lashed so as to conform to safety requirements. The layout is not critical.

The power supply is a mains operated one giving $7.5 \mathrm{~V}$ at $0.5 \mathrm{~A}$.

\section{DECODER COMPONENTS}

The cost of components is $£ 40$ (about $\$ 90$ ), and the construction time 40 man-hours. All the resistors and the components associated with the NE555V are as described in the recorder section. The three $10-\mathrm{nF}$ capacitors associated with the CD4046AEs are $21 / 2 \%$ polystyrene.

\section{PERFORMANCE ON THE TEST}

As a preliminary step towards performance norms for this task, five enlisted men were required to carry out 20 -min continuous responding both in the present four-choice device and, for comparison, upon the conventional five-choice test of Leonard (1959), for which the four-choice is intended as a portable substitute. Three of the subjects carried out the following routine: $5 \mathrm{~min}$ practice on the four-choice test. $5 \mathrm{~min}$ rest. $20 \mathrm{~min}$ test on the four-choice, leave testing room, return $15 \mathrm{~min}$ later and follow same routine using the five-choice test. The other two 
Table 1

\begin{tabular}{|c|c|c|c|c|c|c|c|c|c|c|c|c|c|}
\hline & \multicolumn{8}{|c|}{ 5-Min Periods } & \multicolumn{5}{|c|}{ First Five 1-Min Periods } \\
\hline & \multicolumn{4}{|c|}{ Five-Choice } & \multicolumn{4}{|c|}{ Four-Choice } & \multicolumn{5}{|c|}{ Four-Choice } \\
\hline & 1 & 2 & 3 & 4 & 1 & 2 & 3 & 4 & 1 & 2 & 3 & 4 & 5 \\
\hline Average RT (msec) & 771 & 765 & 771 & 741 & 571 & 581 & 583 & 587 & 544 & 575 & 557 & 580 & 607 \\
\hline Average Gaps & 20.0 & 25.2 & 26.4 & 27.2 & 25.2 & 24.0 & 28.8 & 29.2 & 2.4 & 3.2 & 2.0 & 3.6 & 4.8 \\
\hline Average Errors & 38.8 & 49.2 & 51.2 & 34.8 & 22.8 & 26.0 & 21.6 & 26.8 & 5.2 & 10.0 & 8.8 & 7.6 & 7.2 \\
\hline
\end{tabular}

subjects followed the same procedure but with the five-choice test first and the four-choice second.

Table 1 shows mean reaction times (RTs), gaps, and errors in each 5-min period of the test for both the new four-choice and the old five-choice tests. Gaps for the five-choice were, as usual, counted whenever the subject allowed an interval of 1.5 sed or more to elapse between responses, a period of $3 \mathrm{sec}$ for example scoring 2 gaps. In the four-choice, gaps were taken as the total number of response intervals of $1 \mathrm{sec}$ or longer, a cutoff point which gave approximately equal numbers of gaps to those occurring in the five-choice.

Classically, choice serial reaction time tasks of the five-choice type are known to show the effect of prolonged work by an increase in the number of abnormally long response times, called "blocks" in the work of Bills (1931), and later called "gaps" with specific reference to Leonard's five-choice test (see summary review by Poulton, 1971). During the course of the five-choice test in the present trials, it was again gaps which showed a decline in performance, the index of this within-test deterioration (WTD) being taken as second half score minus first half score. Neither RT nor errors showed a similar decline in the five-choice. When the subjects were ranked for WTD on the basis of these scores, the rankings did not correlate positively with the corresponding rankings for gaps; indeed, the correlation between gaps and errors was negative (Spearman's $r=-0.87, \mathrm{p}<.06$, two-tail level in this case, one-tail in all significance tests that follow).

In the four-choice test, by contrast, all three scores agreed reasonably well with each other in reflecting a general deterioration in performance during the test, the most consistent fall being that in RT. The scores also agreed in reflecting individual WTDs, the relevant correlations being: RT vs. gaps, $r=+0.90(p<.02)$; RT vs. errors, $r=+0.83$ ( $\mathrm{p}<.05$ ); gaps vs. errors, $r$ $=+0.88(\mathrm{p}<.025)$. Kendall's concordance measure across individuals between the three WTD scores was $0.844(p<.01)$. Thus, the four-choice appears to have considerable internal consistency, and more than the five-choice.

We must now determine whether the four-choice adequately reflects the classical fatigue-sensitive score of the five-choice, namely gaps. Subjects were ranked on the basis of their WTD in five-choice gaps, and these rankings were correlated with similar ones for four-choice parameters of WTD as follows: five-choice gaps vs. four-choice RT, $r=+0.95$ $(p<.01)$; vs. four-choice gaps, $r=+0.82(p<.05)$; and vs. four-choice errors, $r=+0.84(p<.05)$. It seems reasonable, therefore, to accept these results as a preliminary indication that the four-choice fulfills its primary requirement of reflecting fatigue due to prolonged work in the same way as gaps do in the classical but unwieldy five-choice.

Table 1 also shows mean RT, gaps, and errors in the four-choice for each of the first five 1 -min periods of the test. Performance declined during the first 5 min of the test in both RT and gaps. RT during the 4 th and 5th minutes minus RT during the 1 st and 2 nd minutes was taken as the best index of this decline and was correlated as before across subjects with the whole test WTD in five-choice gaps $(r=+0.75$, n.s.), four-choice RT $(r=+0.84, p<.05)$ four-choice gaps $(r=+0.98, p<.01)$, and four-choice errors $(r=+0.91, p<.05)$. Although the claim must remain tentative until checked by more exhaustive comparisons of four- and five-choice tests, particularly under conditions of environmental stress, it appears possible that $5 \mathrm{~min}$ of testing on the four-choice may suffice to show effects upon RT of continuous repetitive responding which would only become manifest on the five-choice with some $20 \mathrm{~min}$ of work.

\section{REFERENCE NOTE}

1. Leonard, J. A. 5-choice serial reaction apparatus. Applied Psychology Research Unit, Report No. 326/59, Cambridge, England, 1959.

\section{REFERENCES}

Bicls. A. G. Glocking: A new principle in mental fatigue. American Journal of Psychology, 1931, 43, 230-245.

Poulton, E. C. Environment and human efficiency. Springfield, Ill: Thomas, 1971.

Wilkinson, R. T. Some factors influencing the effect of environmental stressors on performance. Psychological Bulletin, 1969, 72. 260-272.

\section{NoTES}

1. In both circuit diagrams, the convention adopted with respect to resistors and capacitors has been that of using the denomination in place of the decimal point, e.g., $2 \mathrm{~K} 2$ instead of $2.2 \mathrm{~K} \mathrm{ohm}$. This was done in order to achieve compact diagrams without the chance of mistakenly omitting decimal points. Also, the term "nf" (nanofarads) is used in place of $1,000 \mathrm{pF}$ (picofarads).

2. Hamlin $1 \mathrm{~KB}$ switches can be obtained from Hamlin Inc., Lake and Grove Streets, Lake Mills, Wisconsin 53551. 